\title{
SIMULATION NUMERIQUE D'UNE HOULE DE CANAL
}

\author{
C. Bertelle ; J.-F. Lhuissier \\ Laboratoire de Mécanique des Fluides - Université du Havre \\ 25, rue Ph. Lebon, B.P. 540, 75058 Le Havre
}

\begin{abstract}
:
Numerical simulation of a wave tank

A wave tank numerical simulation is presented. This code solves the Navier-Stokes equations in velocity-pressure form, using finite volumes techniques. A second order spatial discretization method and implicit scheme for the transient problem resolution are used. A structured, moving mesh allowed to accuratly follow the free surface motion.

Kinematic fields computed from this code are shown, concerning swell and standing wave flows. The code is validate on comparisons with data obtained in laboratory wave channel. Some results are finally presented concerning flows around horizontal plane plates. Vorticity areas appear depending on choosen viscosity.
\end{abstract}

\section{INTRODUCTION}

La houle a souvent été abordée à partir de théories potentielles ([3], [5], par exemple), alors que son approche, grâce à des simulations numériques basées sur la résolution des équations Navier-Stokes, est encore assez peu fréquente. Parmi celle-ci, on notera, toutefois, le NSL du LNH/EDF (Daubert, Cahouet [4]) et une résolution en fonction du courant-tourbillon formulée par Chagdali [2].

Notre motivation a été de fournir un outil numérique permettant de compléter des études expérimentales par Vélocimétrie Doppler Laser effectuées au Laboratoire de Mécanique des Fluides du Havre. Ces travaux expérimentaux font apparaître des phénomènes dus aux effets de viscosité du fluide ([1], [8], [9]). 


\section{DESCRIPTION DU PROBLEME PHYSIQUE}

On se propose de simuler un écoulement de houle généré par un batteur plan, dans un canal ouvert. Une vue schématique du domaine physique considéré est donnée par la figure 1.

Le domaine est délimité par trois frontières physiques :

- un batteur piston ou incliné dont le déplacement est périodique et sinusoïdal en temps ;

- un fond horizontal fixe ;

- une surface libre se déplaçant en suivant le mouvement du fluide.

Le domaine n'est pas borné sur le côté latéral opposé au batteur.

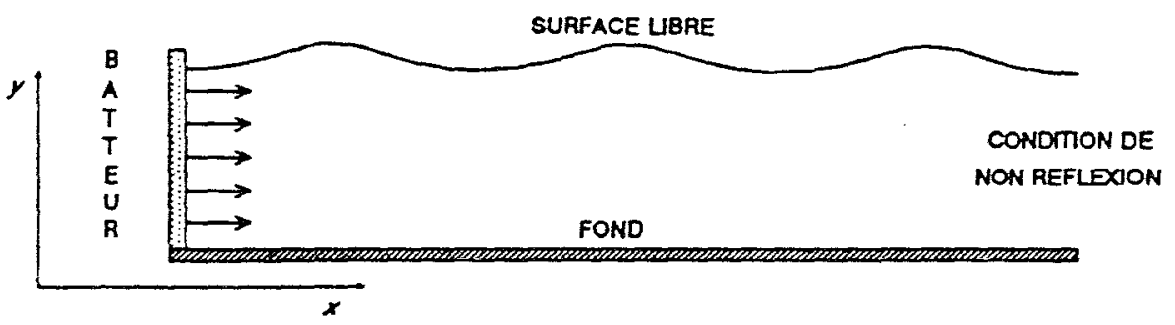

fig. 1 : domaine d'étude

\section{EQUATIONS FONDAMENTALES :}

On suppose que l'écoulement instationnaire est bidimentionnel. Il est gouverné par les équations de Navier-Stokes :

- équation de conservation de la masse :

(1) $\operatorname{div} u=0$

- équation de conservation de la quantité de mouvement :

(2) $\overrightarrow{\mathrm{Du}}=-\overrightarrow{\operatorname{Drad}}\left(\frac{\mathrm{P}}{\rho}\right)+\overrightarrow{v \Delta \mathrm{u}}+\overrightarrow{\mathrm{F}}$ 
où $\vec{F}$ correspond aux forces extérieures, dans notre cas, ce sont les forces de gravité.

Les vitesses sont nulles sur le fond et sont déterminées par la cinématique du batteur sur la frontière latérale gauche. Pour ces deux frontières, la pression vérifie l'équation de conservation de quantité de mouvement dont l'écriture se trouve simplifiée par la connaissancé des vitesses et par l'utilisation de la conservation de la masse.

A la surface libre, la pression est atmosphérique et les dérivées normales des vitesses s'annuleñt.

Sur la frontière latérale droite, on recherche une condition permettant à l'onde de quitier le domaine sans créer de réflexion. La célérité de l'onde sortante $c$ est calculée grâce aux déformations de la surface libre générées. Chacune des variables calculées $\varphi$ satisfait à l'équation d'onde (condition de radiation de Sommerfeld [12]) :

$\frac{\delta \psi}{\delta t}=-c \frac{\delta \psi}{\delta x}$

\section{ALGORITHME DE CALCUL}

Les équations d'évolution non linéaires précédentes sont écrites sous forme conservarrice et sont traitées dans le schéma itératif suivant :

1) détermination de la première composante de vitesse u grâce à l'équation de conservation de quantité de mouvement :

(4) $\frac{\delta u}{\delta t}+\frac{\delta}{\delta x}\left(u^{2}-v \frac{\delta u}{\delta x}\right)+\frac{\delta}{\delta y}\left(u v-v \frac{\delta u}{\delta y}\right)+\frac{\delta}{\delta x}\left(\frac{P}{\rho}\right)=0$

2) détermination de la deuxième composante de vitesse $v$ grâce à l'équation de conservation de la masse :

$\frac{\delta v}{\delta y}=-\frac{\delta u}{\delta x}$ 
3) détermination de l'élévation d'eau $H$ grâce à l'équation :

(6) $\frac{\delta H}{\delta t}=v-u \frac{\delta H}{\delta x}$

5) détermination de la pression $P$ grâce à l'équation de Poisson :

$$
\Delta(\mathrm{P} / \rho)=2\left(\frac{\delta \mathrm{u}}{\delta \mathrm{x}} \frac{\delta \mathrm{v}}{\delta \mathrm{y}}-\frac{\delta \mathrm{u}}{\delta \mathrm{y}} \frac{\delta \mathrm{v}}{\delta \mathrm{x}}\right)
$$

\section{DISCRETISATION DU DOMAINE DES EQUATIONS :}

Le domaine de calcul est discrétisé en cellules, composant un maillage structuré et déformable, qui suit l'évolution de la surface libre. Les différentes variables de calcul sont situées sur une grille décalée, fréquemment utilisée, par exemple, dans les méthodes des volumes finis ([6], [13]).

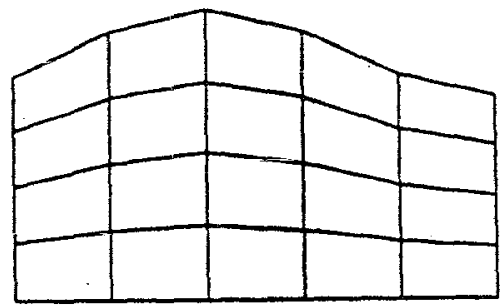

fig. 2 : maillage deformadie

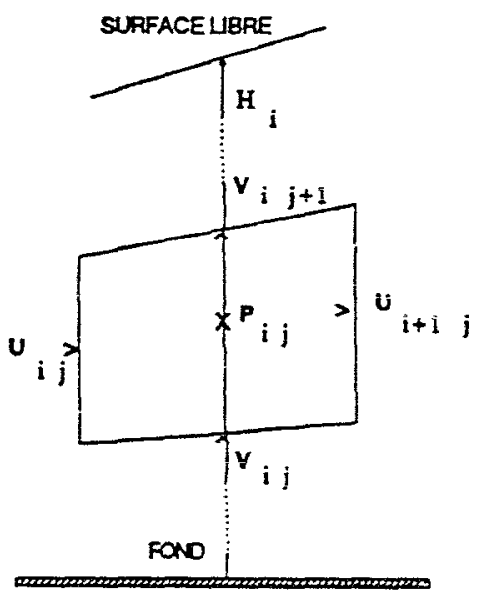

fig. 3 : grille décalée

La discrétisation des équations (4)-(7) est basée sur un schéma hybride de volume finis (on pourra se référer à Patankar [14]). On les intègre sur chacune des cellules $\Omega$ qui composent le maillage. 


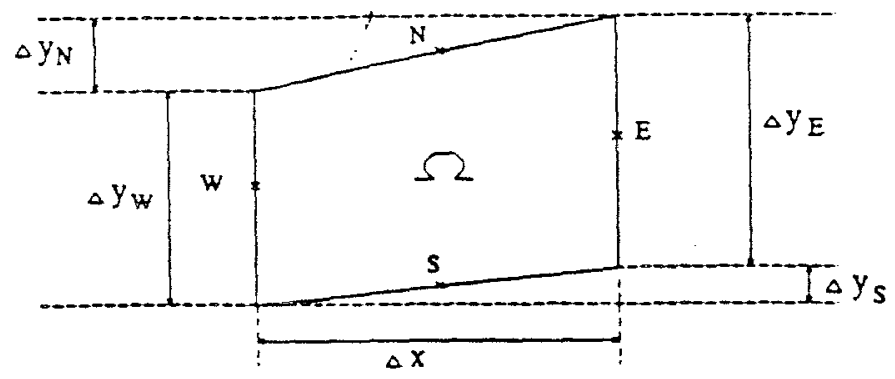

fig. 4 : géométrie dune cellule

Les termes des équations aux dérivées partielles (4)-(7) seront intégrés sur chaque cellule suivant leur nanure :

- les termes dérivés suivant la direction verticale s'intègrent et se discrétisent suivant :

$\int_{\Omega} \frac{\delta \gamma}{\delta y} \mathrm{~d} \Omega=\Delta \mathrm{x}\left(\gamma_{\mathrm{N}}-\gamma_{\mathrm{S}}\right)$

où $\gamma_{N}$ et $\gamma_{S}$ représentent les valeurs de $\gamma$ sur les centres des faces Nord et Sud;

- les termes dérivés suivant la direction horizontale s'intègrent et se discrétisent suivant :

$\int_{(9)} \frac{\delta \beta}{\delta x} \mathrm{~d} \Omega=\beta_{E} \Delta y_{E}-\beta_{w} \Delta y_{w}+\beta_{s} \Delta y_{S}-\beta_{N} \Delta y_{N}$

où $\beta_{N}, \beta_{S}, \beta_{W}$ et $\beta_{E}$ représentent les valeurs de sur les centres des faces Nord, Sud, Ouest et Est ;

- pour les termes dérivés par rapport au temps, l'intégration se fera en utilisant le théorème de Transport :

$\int \frac{\delta \alpha}{\delta t} \mathrm{~d} \Omega=\frac{D}{D t} \int \alpha \mathrm{d} \Omega-\int \alpha \mathrm{V}_{\mathrm{F}}$. 
où $\delta \Omega(t)$ est le contour de $\Omega(t)$.

$$
\overrightarrow{\mathrm{V}_{\mathrm{F}}} \text { et } \overrightarrow{\mathrm{n}}
$$

sont respectivement la vitesse et la normale des faces de la cellule.

La discrétisation complète des équations conduit à un calcul direct en $\mathrm{v}$, un schéma explicite en temps pour $\mathrm{H}$ et des résolutions de systèmes linéaires pentadiagonaux pour u et $P / P$. Ces derniers sont résolus itérativement en deux étapes par un algorithmeTDMA en séparant les directions d'espace.

\section{EXEMPLES DE CALCULS ET VALIDATIONS EXPERI- MENTALES}

Sur la figure 5, on a représenté les champs cinématiques obtenus par le code de calcul pour une longueur d'onde de houle de 1,4 m et une profondeur d'eau, au repos, de $0,27 \mathrm{~m}$. Ilc correspondent :

- pour la partie A : à un écoulement caractéristique de houle obtenu en prenant la condition de radiation de Sommerfeld sur la frontière latérale droite ;

- pour la partie B : à un écoulement caractéristique de clapotis, obtenu en prenant une condition de réflexion sur la frontière latérale droite (on considère alors que cette frontière est une paroi où les vitesses s'annulent par adhérence du fluide).
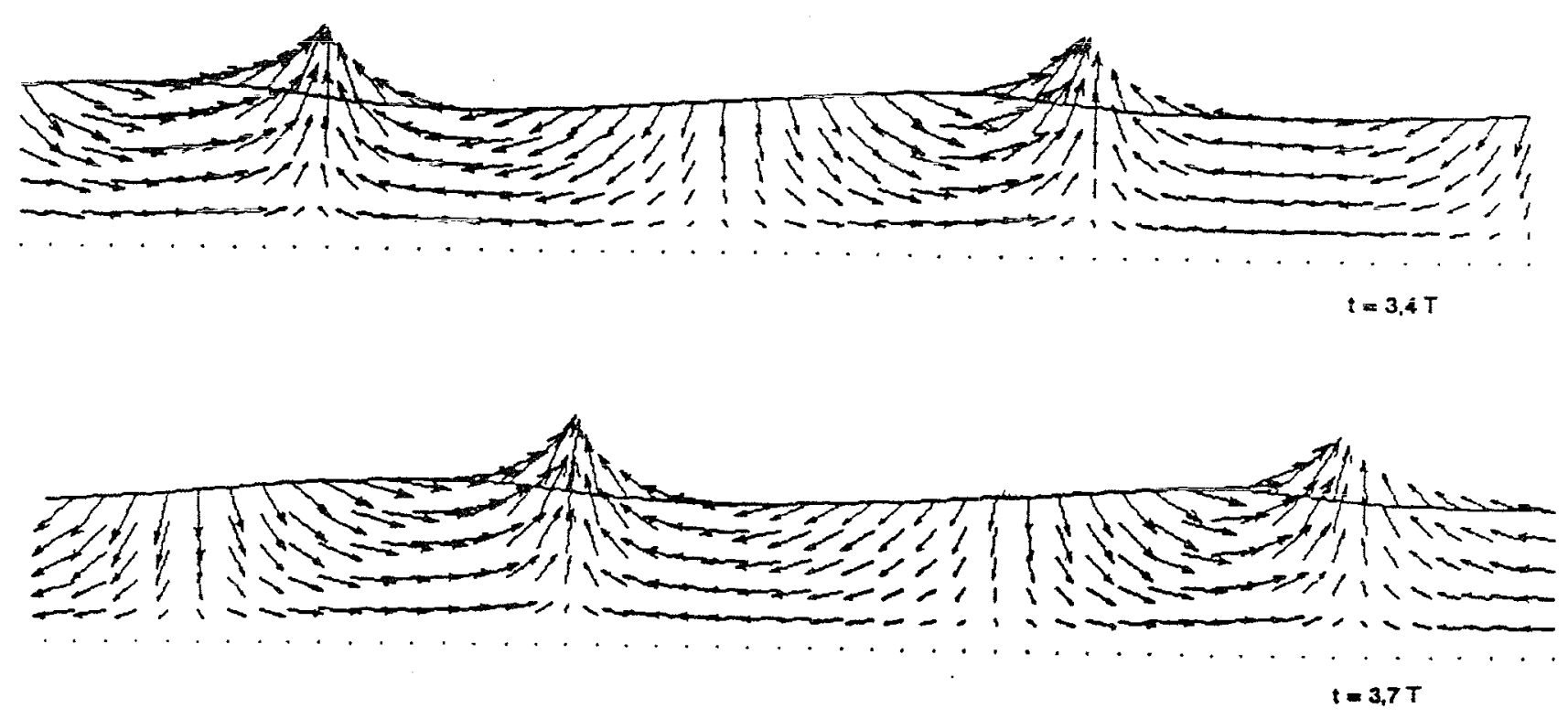

A - houle 

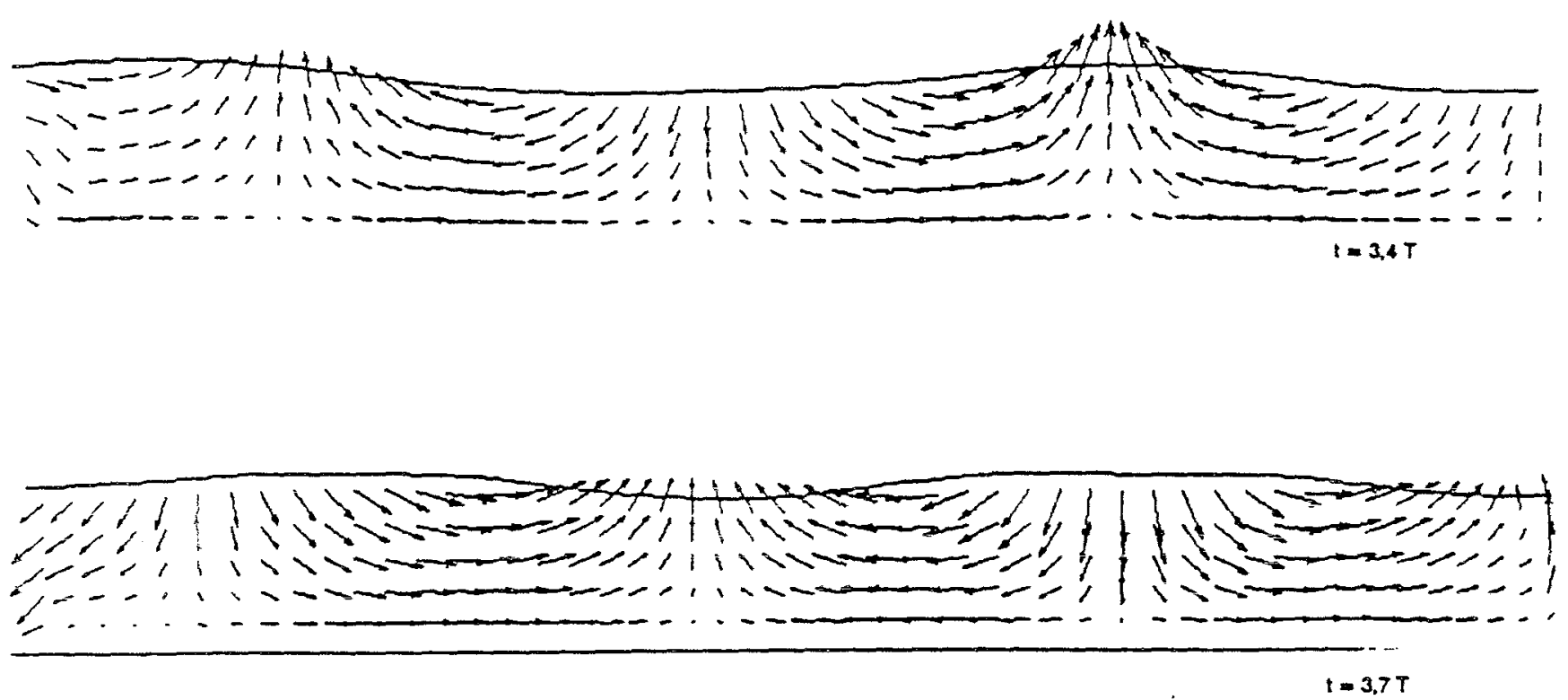

B - clapotis

fig. 5 champs cinématiques ơune houle et doun dapotis

La figure 6 permet de comparer des résultats de calcul avec des mesures effectuées par Vélocimétrie Doppler Laser par Jarno [7]. Le canal à houle expérimental mesure $9 \mathrm{~m}$ et le niveau d'eau au repos est de $0,27 \mathrm{~m}$.La période du batteur est de $1,03 \mathrm{~s}$. Les champs de vitesses présentés correspondent à des profils de la composante horizontale à différentes phases de la houle (indiquée en degré en haut de chaque courbe) et situés approximativement au centre du canal.

Les résultats sont, pour l'essentiel, en accord avec les théories de Miche [10] sur les houles en eau peu profonde. Des différences par rapport à cette théorie se retrouvent à la fois sur les calculs et sur les résultats :

- le gradient de vitesse (en valeur absolue) sur la surface libre augmente au sommet de la vague et diminue au creux;

- les vitesses au fond sont plus grandes au sommet qu'au creux, ce qui laisse présumer d'un transport de masse ;

- le changement d'inclinaison des profils vers le sens décroissant s'effectue bien avant l'annulation des vitesses sur le fond (entre $40^{\circ}$ et $50^{\circ}$ ). 


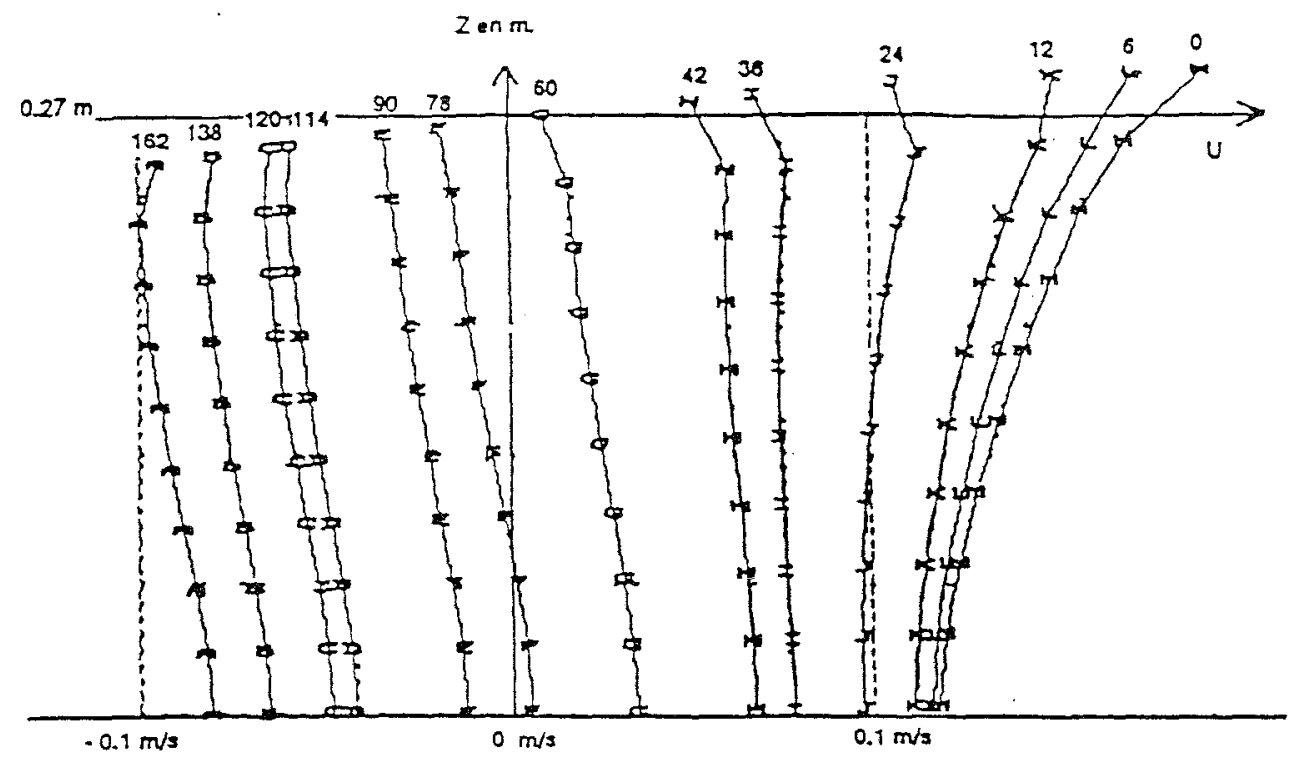

A - résultats de calcul

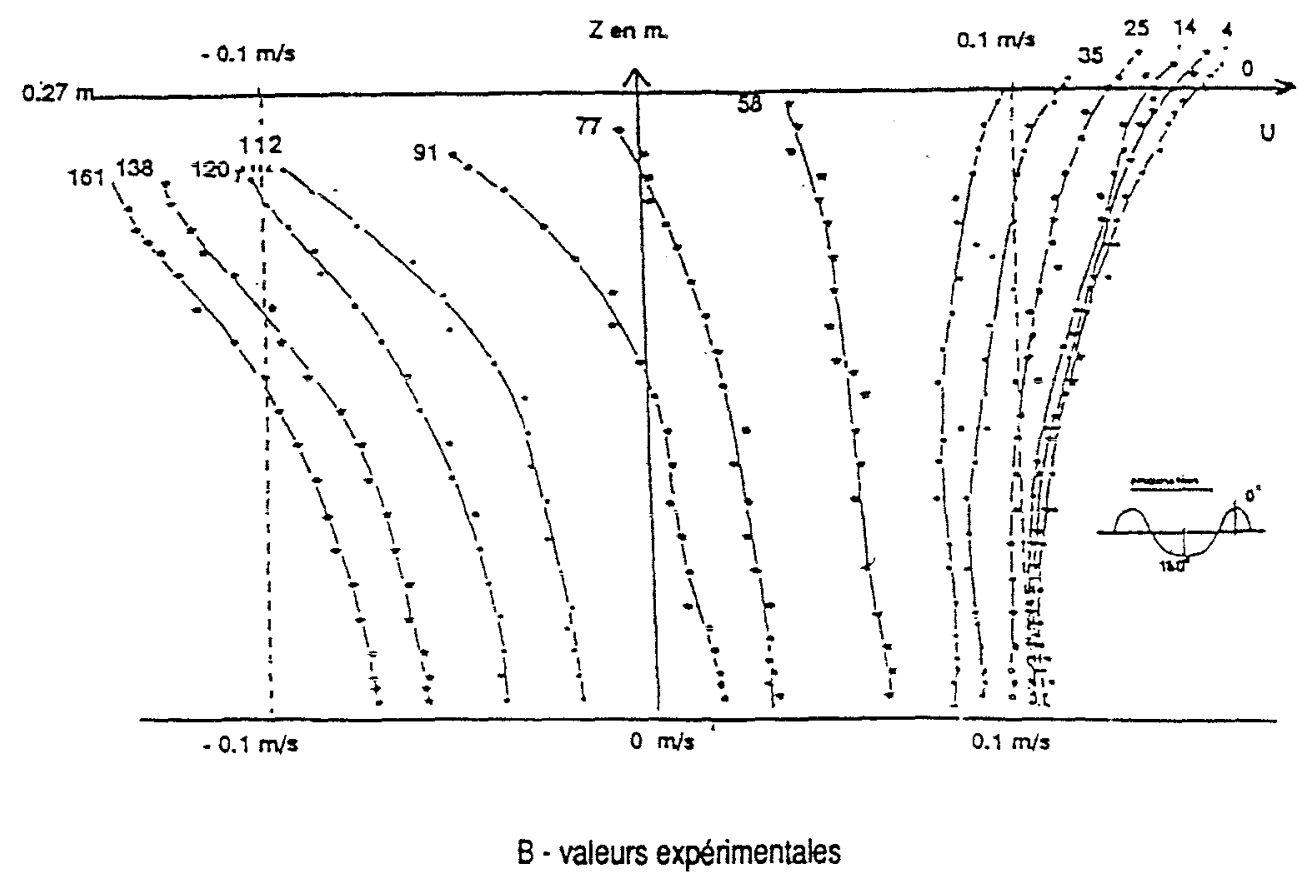

fig. 6 : comparaison des profils de vitesses horizontales en eau peu proionde 

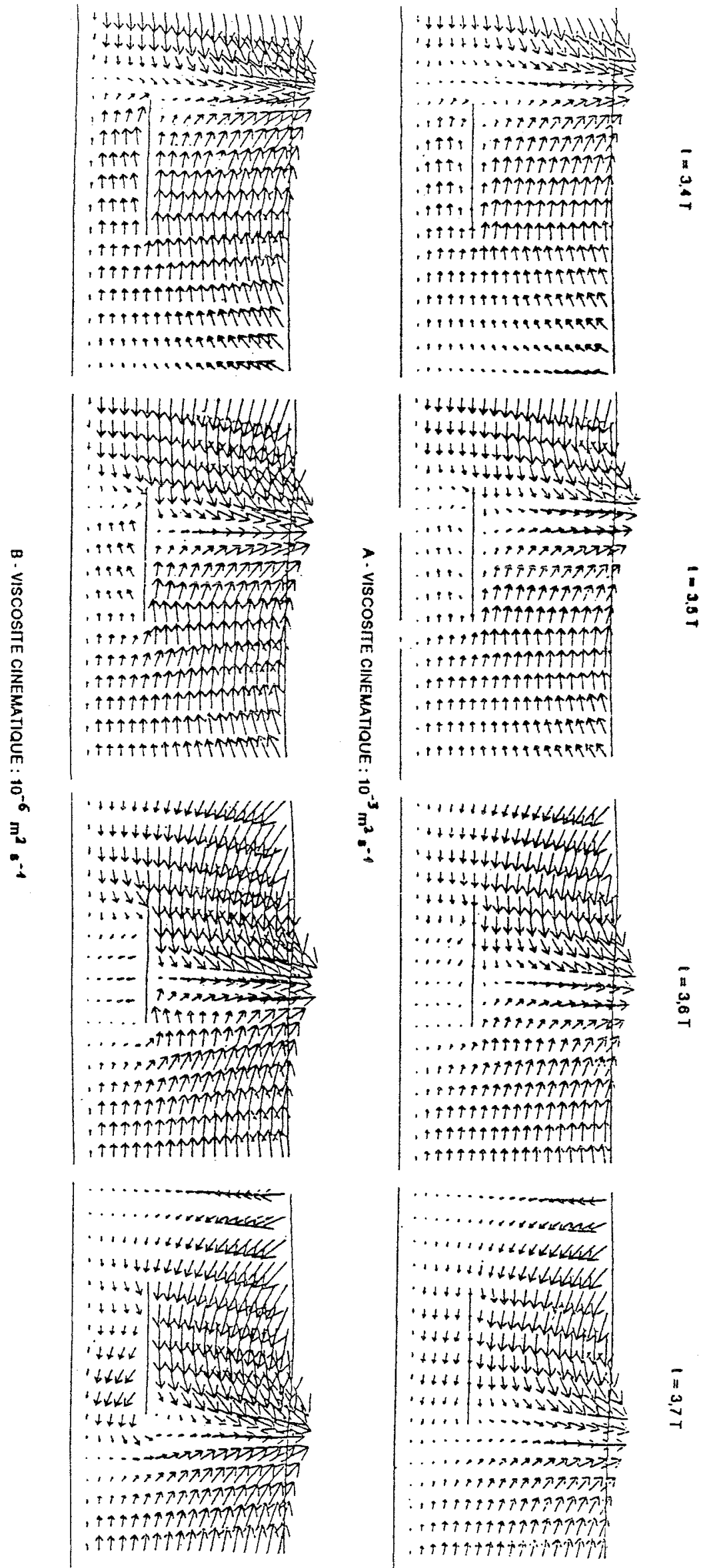

fig 7 : ecoulement autour dune plaque 
Sur la figure 7, on montre les premiers essais de calcul effectués autour d'obstacles immergés : il s'agit ici d'une plaque plane horizontale immergée dont la longueur est du quart de la longueur d'onde de la houle. Par adhérence, la vitesse du fluide est supposée nulle sur la plaque. Afin de faire ressortir les effets dus à la viscosité, on a effectué des simulations avec des viscosités dynamiques différentes : $10^{-3}$ et $10^{-6} \mathrm{~m}^{2} \mathrm{~s}^{-1}$. On observe, dans le premier cas, une zone de création de rotationnel au bord de fuite amont à 3,4 périodes qui est due à une dépression engendrée par les frottements visqueux sur la plaque Dans le deuxième cas, on voit se former des incurvations des lignes de courant au bord de fuite aval, à 3,5 et 3,6 périodes. Elles correspondent à la création d'une couche limite qui se décolle à 3,7 périodes, au passage du sommet de la vague. Une zone de rotationnel sur le bord de fuite aval apparait à cet instant. Ce demier phénomène a été étudié expérimentalement par Brossard [1] et Rivoalen [15]. Néanmoins, le décollement a été observé beaucoup plus tôt. Cet écart peut être expliqué par un manque de résolution spatial dans notre calcul.

\section{REFERENCES}

[1] BROSSARD : Contribution d̀ l'étude de la turbulence d travers l'analyse d'un écoulement généré par la houle entre deux plaques, Thèse de 3ème Cycle de Rouen, 1986.

[2] CHAGDALI : Simulation numérique d'écoulement à surface libre d'un canal a houle, Thèse d'Etat à Casablanca, 1990.

[3] COINTE : Queiques aspects àe la simuiation numérique d'un canal à houle, Thèse des Ponts et Chaussées, 1989.

[4] DAUBERT ; CAHOUET : "Approche numérique de la houle par les équations de Navier-Stokes", Annales des Ponts et Chaussées, 1984.

[5] GUEVEL ; LANDEL ; BOUCHET ; MANZONE : "Le phénomène d'un mur d'eau oscillant et son application pour protéger un site côtier soumis à l'action de la houle", PIANC-AIPCN, $\mathrm{n}^{\circ}$ 52, 1986.

[6] HARLOW ; WELCH : "Numérical calculation of time dependant viscous incompressible flow or fluid with free surface", Physics of Fluids, vol. 8, $\mathrm{n}^{\circ}$ 12, p. 2182-2189, 1965.

[7] JARNO : Analyse de la cinématique de la houle en eau peu profonde, Rapport de DEA, Le Havre, 1986. 
[8] JARNO ; BERTELLE ; BELORGEY : "Dynamic influence of waves on a horize cal cylinder beneath waves", Proc. Int. Congress on Computer Modeng in Ocean Ing., Schrefler and Zienkiewicz (eds), Balkema, 1988, p. $60 \% 615$.

[9] LEBAS : Etude de la couche limite générée par les vagues sur le fonds d'un canal d houle, Thèse d'Etat du Havre, 1986.

[10] MICHE : "Mouvements ondulatoires de la mer en profondeur constante ou décroissante", Annales des Ponts et Chaussées, 1944.

[11] NICHOLS ; HIRT ; HOTCHKISS : SOLA-VOLF : a solution algorithm for transiant fluid flow with multiple free boudaries, Los Alamos Scientific Laboratory repon LA-8355, August 1980.

[12] ORLANSKI : "A simple boundary conditions for unbouded hyperbolic flows", J. of Comput. Phys., 21, p. 251-269, 1976.

[13] PATANKAR ; SPALDING : "A calculation procedure for heat, mass and momentum transfer in three-dimensional parabolic flows", Int. J. Heat Mass Transfer, vol. 15, p. 1787-1806, 1972.

[14] PATANKAR : Numerical heat transfer and fluid flow, Hemisphere pub. corp, Mc Graw-Hill book company, New-York, 1980.

[15]RIVOALEN : Evolution de structures tourbillonnaires générées par un canal d houle, Rapport de DEA, Le Havre, 1989. 